\title{
Socio-demographic predictors of gender inequality among heterosexual couples expecting a child in south-central Uganda
}

\author{
Caroline J Vrana-Diaz ${ }^{1}$, Jeffrey E Korte ${ }^{1}$, Mulugeta Gebregziabher ${ }^{1}$, Lauren Richey ${ }^{2}$, \\ Anbesaw Selassie ${ }^{1}$, Michael Sweat ${ }^{3}$, Harriet Chemusto ${ }^{4}$, Rhoda Wanyenze ${ }^{5}$
}

1. Department of Public Health Sciences, Medical University of South Carolina, 135 Cannon Street, Suite 303, Charleston, SC, USA 29425.

2. Section of Infectious Disease, Department of Medicine, Louisiana State University Health Sciences Center, 1542 Tulane Avenue, Suite 331, New Orleans, LA 70112.

3. Department of Psychiatry and Behavioral Sciences, Medical University of South Carolina, 176 Croghan Spur Road, Suite 104, Charleston, SC 29407.

4. Mildmay Uganda, P.O. Box 24985, Kampala, Uganda.

5. School of Public Health, College of Health Sciences, Makerere University, New Mulago Hill Road, Mulago, Kampala, Uganda.

\begin{abstract}
:
Background: Gender inequality is a pervasive problem in sub-Saharan Africa, and has negative effects on health and development.

Objective: Here, we sought to identify socioeconomic predictors of gender inequality (measured by low decision-making power and high acceptance of intimate partner violence) within heterosexual couples expecting a child in south-central Uganda.

Method: We used data from a two-arm cluster randomized controlled HIV self-testing intervention trial conducted in three antenatal clinics in south-central Uganda among 1,618 enrolled women and 1,198 male partners. Analysis included Cochran Mantel-Haenzel, proportional odds models, logistic regression, and generalized linear mixed model framework to account for site-level clustering.

Results: Overall, we found that $31.1 \%$ of men had high acceptance of IPV, and $15.9 \%$ of women had low decision-making power. We found religion, education, HIV status, age, and marital status to significantly predict gender equality. Specifically, we observed lower gender equality among Catholics, those with lower education, those who were married, HIV positive women, and older women.

Conclusion: By better understanding the prevalence and predictors of gender inequality, this knowledge will allow us to better target interventions (increasing education, reducing HIV prevalence in women, targeting interventions different religions and married couples) to decrease inequalities and improve health care delivery to underserved populations in Uganda. Keywords: Gender inequality, Pregnancy, HIV/AIDS, Prenatal Care, Uganda.

DOI: https://dx.doi.org/10.4314/ahs.v20i3.23

Cite as: Vrana-Diaz CJ, Korte JE, Gebregziabher M, Richey L, Selassie A, Sweat M, et al. Socio-demographic predictors of gender inequality among heterosexual couples expecting a child in south-central Uganda. Afri Health Sci. 2020;20(3): 1196-1205. https://dx.doi. org/10.4314/abs.v20i3.23

\section{Introduction}

Gender equality refers to the equal rights, responsibilities and opportunities of women and men, and equality between women and men is both a human rights issue and is necessary for sustainable development ${ }^{1}$.
Corresponding author:
Caroline J Vrana-Diaz,
135 Cannon Street, 305 W (Q), Charleston,
SC, 29524, (717) 364-8097.
ORCID ID: 0000-0001-9037-2761
Email:vrana@musc.edu

However, in most sub-Saharan African societies, men have substantial power over women, and many national achievements in reproductive health, empowerment, and labor market participation have been diminished by gender inequality ${ }^{2,3}$.

There have been many studies in sub-Saharan Africa assessing sociodemographic factors and gender inequality, which have shown that low educational, occupational, and economic opportunities, and food insecurity have been associated with gender inequality, although with differing measures of gender inequality ${ }^{4-13}$.
\end{abstract}


Pregnant women are especially important to study, as they are considered a vulnerable population, and gender inequality affects the ability of pregnant women to attain reproductive health services, and maternal and child health outcomes. There has been previous work showing the associations between women's decision-making power and autonomy, and how high decision-making autonomy and lack of intimate partner violence is associated with beneficial maternal and child health outcomes ${ }^{14-18}$. However, there has been a relative lack of studies assessing sociodemographic variables associated with gender inequality within the context of a pregnancy. We recently performed an analysis of social and economic variables and gender inequality among heterosexual couples expecting a child in central Kenya, which had some promising results and areas to target for improvement of these gender inequality measures ${ }^{19}$. Therefore, we attempted to identify potential associations between social and economic variables and gender inequality (which in this case were measured as high acceptance of intimate partner violence and low women's decision-making power), within the context of heterosexual couples expecting a child in south-central Uganda.

\section{Methods}

\section{Design and Study Population}

These data stem from a two-arm cluster randomized HIV self-testing intervention trial conducted in three clinics in south-central Uganda, with study information collected at baseline, and both a one-month and threemonth follow-up visit. Briefly, women were eligible to participate in the study if they were currently pregnant, at least 14 years old (pregnant women between 14 and 18 years old are considered emancipated minors in Uganda), and attending an antenatal clinic (ANC) for this pregnancy. Furthermore, the women were eligible if they had reported contact (either sexual or otherwise) with their male partner at least once per week, if their male partner was either HIV negative or their status unknown at the time of the woman's recruitment, and that their male partner was at least 18 years of age and had not tested for HIV in the past six months. After the women provided informed consent, they were randomized by clinic day into one of two arms: Arm 1 , the standard of care for antenatal clinics in Uganda, and Arm 2: standard of care plus the provision of OraQuick HIV self-testing (HST) kits with instructions for testing at home. The women also then completed a baseline questionnaire. Both one month and three months after enrollment, the women were interviewed to ascertain whether or not their male partner tested for HIV, and the method of testing. The male partners were also contacted at one month and three months, and those consenting for an interview were administered a questionnaire on socio-demographics and HIV testing history.

\section{Measurements}

Socio-demographic variables included age of both the man and woman (categorized from a continuous variable based on distributional balance), woman's education level, religion, woman's employment status, marital status, and woman's report on the male partner's additional sexual partners. Further variables included partner's education level, food insecurity, partner's employment status, woman's HIV status, and equality in earnings (the proportion of household expenses met by the woman's earnings: none, less than half, half, more than half, or all).

The two primary outcome variables used in this study are measures of gender equality - namely attitudes towards IPV and decision-making power. Attitudes towards IPV was measured by the male's report at month 1 for the validated Violence Domain of the Gender Equitable Scale, a 5 question scale regarding hypothetical violence towards women, with available answers on a 3-point scale, where $1=$ agree, $2=$ partially agree, and $3=$ disagree. Scores across all questions were summed, and categorized into three levels: high acceptance of IPV (score of 5-11), medium acceptance of IPV (score of 12-13), and low acceptance of IPV (score of 1415), where the higher the score, the lower acceptance of IPV (i.e. higher support for gender norms)20. Decision-making power was measured by the woman's report on decision making for: the woman's earnings, the woman's healthcare, major household purchases, daily household needs, and visiting family or relatives, with available answers of: 1) Myself, 2) My partner, 3) Jointly, or 4) Others. Each response to the five questions was dichotomized, with a value of 1 if the woman reports that a decision was made by either herself or jointly, and 0 if the decision was made by her male partner or someone else. We then created an index by summing the five dichotomized responses, with a value of 0 if the woman made none or only 1 decision (low decision-making power), 1 if she made two or three decisions by herself or jointly (medium decision-making power), and 2 if she made either four or all five decisions by herself or jointly (high decision-making power). 


\section{Data analysis}

We summarized data using descriptive statistics where mean/SD were reported for continuous variables and proportions were reported for categorical variables. To make comparisons between groups, we used the Cochran Mantel-Haenzel statistic. We checked the proportional odds assumption using the score test for proportional odds given in logistic regression ${ }^{21}$. For the first set of analysis, we analyzed gender equality as measured by attitudes towards IPV from the man's report (with an ordinal outcome) and we used a logistic regression framework with a cumulative logit to estimate the odds ratios (OR) and corresponding 95\% CI, due to not enough variability in the site-level clustering for a generalized linear mixed model approach. The second set of analyses was gender equality as measured by decision-making power from the woman's report (with an ordinal outcome), with modeling performed with cumulative logit in a generalized linear mixed models (GLMMs) framework to account for site-level clustering22. We chose our final model for each analysis based on a combination of factors including conceptual plausibility, individual variable significance in the model, confounding, and two measures of model fit (Akaike's Information Criterion and -2 Log Likelihood, when appropriate). A two-sided p-value of $<0.05$ for specific variables was used to assess significance of specific variables, as well as 95\% CI not including 1. Proc GLIMMIX and Proc LOGISTIC in SAS 9.4 (SAS Institute, Cary, NC) was used for the modeling analyses.

\section{Ethical approval}

The original trial was approved by both the institutional review board of the Medical University of South Carolina and Makerere University School of Public Health in Kampala, Uganda. Written informed consent was obtained from all participants. The current data analysis was performed on completely de-identified data.

\section{Results}

Table 1 shows the sociodemographic characteristics of the women and their male partners. Overall, 1,618 women were enrolled and randomized into the study (47.7\% in the standard of care arm and $52.3 \%$ in the intervention arm), 1,347 women and 1,198 of their male partners were interviewed at their one-month follow-up visit, and 1,299 women and 1,123 of their male partners were interviewed at their three-month follow-up visit. For the woman, they were on average 25.2 years old, and the majority had a secondary or higher education $(57.7 \%)$, were currently not married $(83.4 \%)$, were currently not aware of their male partner having any other sexual partners $(79.7 \%)$, always had enough food and the types of food they wanted $(55.5 \%)$, had less than half or none of the household expenses met by their own earnings (76.8\%), and were HIV negative (89.7\%). For decision-making power, $15.9 \%$ of the women had low decision-making power, $43.8 \%$ of the women had medium decision-making power, and $40.4 \%$ had high decision-making power. For the men, they were on average 32.2 years old, and the majority had a secondary or higher education (55.0\%), were currently not married $(84.5 \%)$, were mostly Catholic or Other/Not Christian $(28.1 \%$ and $21.6 \%$, respectively), were either employed for wages or self-employed $(21.1 \%$ and $35.8 \%$, respectively), and the vast majority were HIV negative (97.1\%). Overall, $31.1 \%$ of the men showed high acceptance of hypothetical IPV, $28.9 \%$ had moderate acceptance of IPV, and $40.0 \%$ had low acceptance of IPV. For decision-making power, $15.9 \%$ of the women had low decision-making power, $43.8 \%$ of the women had medium decision-making power, and $40.4 \%$ had high decision-making power. 
Table 1: Characteristics of women attending antenatal care at baseline and characteristics of male partners at month 1 in south-central Uganda ${ }^{a}$

\begin{tabular}{|c|c|c|}
\hline Characteristic & $\begin{array}{l}\text { Women } \\
(\mathrm{n}=1,618) \mathrm{n}(\%)\end{array}$ & $\begin{array}{l}\text { Male partners } \\
(\mathrm{n}=1,198) \mathrm{n}(\%)\end{array}$ \\
\hline Age (years), mean $+\mathrm{SD}$ & $25.2 \pm 5.5$ & $32.2 \pm 8.1$ \\
\hline Missing & $21(1.3)$ & $19(1.6)$ \\
\hline \multicolumn{3}{|l|}{ Age categories } \\
\hline 15-19 (women), 18-24 (men) & $225(14.1)$ & $183(15.5)$ \\
\hline 20-24 (women), 25-34 (men) & $611(38.3)$ & $603(51.1)$ \\
\hline 25-29 (women), 35-44 (men) & $425(26.6)$ & $294(25.0)$ \\
\hline 30-49 (women), $45-68$ (men) & $336(21.0)$ & $99(8.4)$ \\
\hline Missing & $21(1.3)$ & $19(1.6)$ \\
\hline \multicolumn{3}{|l|}{ Level of education } \\
\hline Primary or Lower & $673(42.3)$ & $535(45.0)$ \\
\hline Secondary or Higher & $918(57.7)$ & $655(55.0)$ \\
\hline Missing & $27(1.7)$ & $8(0.7)$ \\
\hline \multicolumn{3}{|l|}{ Religion } \\
\hline Catholic & $181(11.2)$ & $337(28.1)$ \\
\hline Protestant/other Christian & $104(6.4)$ & $219(18.3)$ \\
\hline Other & $152(9.4)$ & $259(21.6)$ \\
\hline Missing & $1181(73.0)$ & $383(32.0)$ \\
\hline \multicolumn{3}{|l|}{ Employment status } \\
\hline Employed for wages & $55(3.4)$ & $253(21.1)$ \\
\hline Self-employed & $112(6.9)$ & $429(35.8)$ \\
\hline $\begin{array}{l}\text { Other (business partnership, unemployed, } \\
\text { student, housewife, retired, other) }\end{array}$ & $272(16.8)$ & $129(10.8)$ \\
\hline Missing & $1179(72.9)$ & $387(32.3)$ \\
\hline \multicolumn{3}{|l|}{ Marital status } \\
\hline Currently married & $263(16.6)$ & $185(15.5)$ \\
\hline Not Married & $1325(83.4)$ & $1005(84.5)$ \\
\hline Missing & $30(1.9)$ & $8(0.7)$ \\
\hline \multicolumn{3}{|l|}{ Male Partner has other sexual partners } \\
\hline Yes & $324(20.3)$ & - \\
\hline No/Don't Know & $1269(79.7)$ & - \\
\hline Missing & $25(1.5)$ & \\
\hline \multicolumn{3}{|l|}{ Food Insecurity } \\
\hline $\begin{array}{l}\text { Always have enough food and the kinds we } \\
\text { want }\end{array}$ & $884(55.5)$ & - \\
\hline $\begin{array}{l}\text { Have enough food, but not always the kinds we } \\
\text { want }\end{array}$ & $608(38.2)$ & - \\
\hline Sometimes don't have enough to eat & $67(4.2)$ & - \\
\hline Often don't have enough to eat & $34(2.1)$ & - \\
\hline Missing & $25(1.5)$ & \\
\hline \multicolumn{3}{|l|}{ Proportion of expenses met by woman's earnings } \\
\hline None & $729(45.8)$ & - \\
\hline Less than half & $501(31.5)$ & - \\
\hline Half & $179(11.3)$ & - \\
\hline More than half & $135(8.5)$ & - \\
\hline All & $47(3.0)$ & - \\
\hline Missing & $27(1.7)$ & \\
\hline Missing & & \\
\hline \multicolumn{3}{|l|}{ HIV Status } \\
\hline Positive & $161(10.4)$ & $29(2.9)$ \\
\hline Negative/Indeterminate/Didn't receive results & $1395(89.7)$ & $983(97.1)$ \\
\hline Missing & $62(3.8)$ & \\
\hline \multicolumn{3}{|l|}{ Intervention Arm } \\
\hline Standard of Care & $771(47.7)$ & $559(46.7)$ \\
\hline HIV self-testing kits & $847(52.3)$ & $639(53.3)$ \\
\hline \multicolumn{3}{|l|}{ Health Facility } \\
\hline Nakaseke & $333(20.6)$ & $299(25.0)$ \\
\hline Mpigi & $559(34.6)$ & $433(36.1)$ \\
\hline Entebbe & $726(44.9)$ & $466(38.9)$ \\
\hline
\end{tabular}


Table 2 shows the bivariate analyses between the sociodemographic characteristics and gender equality. We found that acceptance of IPV (based on the Gender Equitable Men scale) was significantly associated with education, religion, and marital status. Specifically, lower scores on the scale (i.e. high acceptance of IPV) were observed among men with primary or lower education, participants reporting Protestant or other Christian re- ligion or Catholics, and participants who were currently not married. On the other hand, decision-making power was associated with age, education, and income equality: specifically, we observed lower decision-making power to be significantly associated with lower woman's age, primary or lower woman's education, primary or lower man's education, and higher proportion of expenses met by the woman's earnings.

Table 2: Bivariate analysis of sociodemographic characteristics of women and male partners and gender equality (attitudes towards IPV and decision-making power)

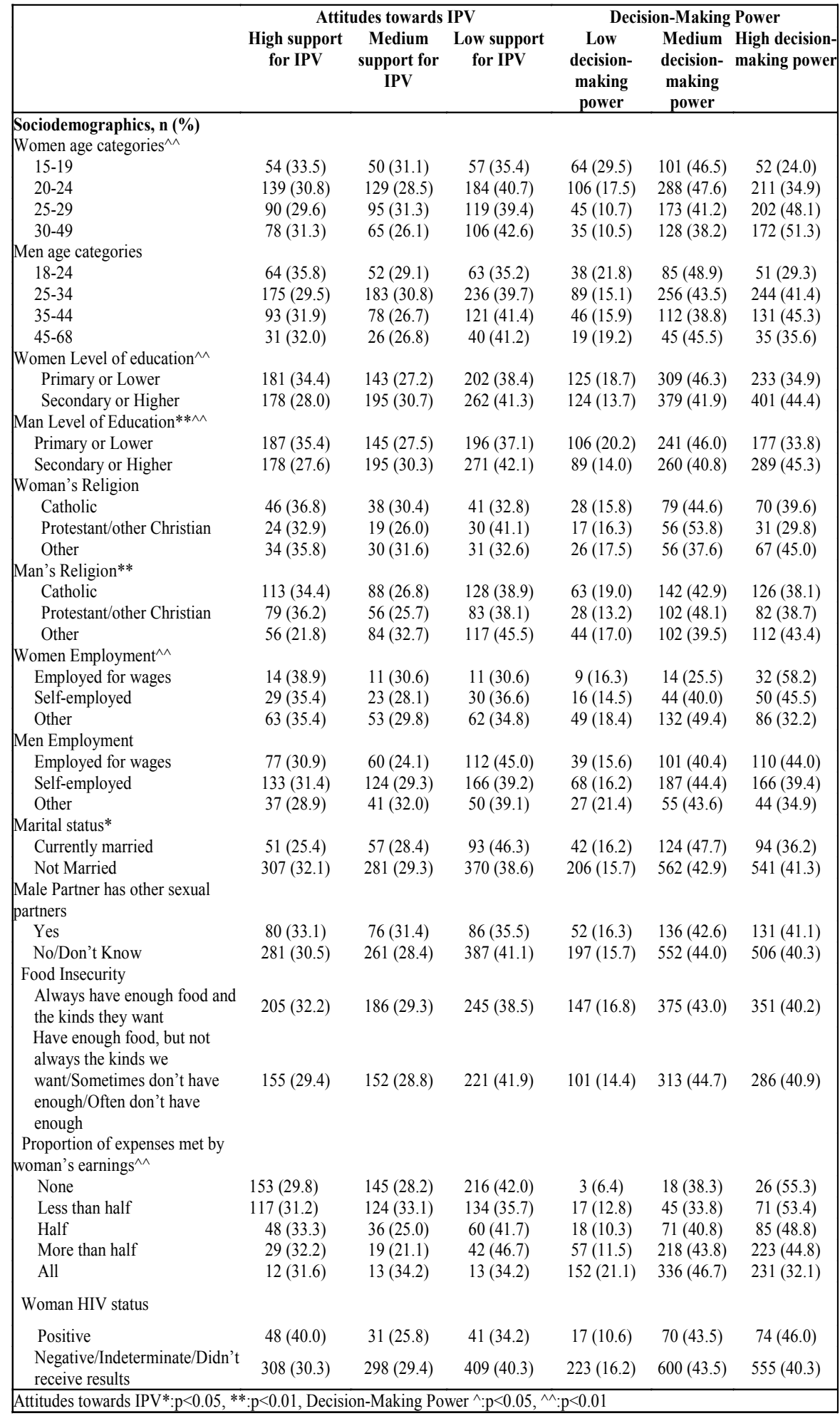


Table 3 shows the modeling of the two gender equality variables by sociodemographics. The significant sociodemographic variables for attitudes towards intimate partner violence included partner's religion, women's education, and woman's baseline HIV status. Specifically, compared to those who were Catholic, men who reported an "Other/Not Christian" religion were less likely to have higher acceptance of IPV (OR 0.65, 95\%
CI 0.47-0.89). Women with a secondary or higher education were less likely to have their partner report increasing acceptance of IPV compared to women with a primary or lower education (OR $0.75,95 \%$ CI 0.560.996). Women who were HIV positive at baseline were more likely to have partner report higher acceptance of IPV compared to women who were HIV negative or unknown HIV status (OR 1.77, 95\% CI 1.19-2.63).

Table 3: Multivariate modeling for the ordinal outcomes of gender inequality

(as measured by attitudes towards IPV and Decision-Making Power)

\begin{tabular}{|c|c|c|}
\hline & $\begin{array}{l}\text { Attitudes Towards Intimate } \\
\text { Partner Violence } \\
\text { OR }(95 \% \text { CI })\end{array}$ & $\begin{array}{c}\text { Decision-Making } \\
\text { Power } \\
\text { OR }(95 \% \text { CI })\end{array}$ \\
\hline \multicolumn{3}{|l|}{ Woman's Age (Ref=18-22) } \\
\hline $23-26$ & $0.99(0.63-1.55)$ & $0.67(0.43-1.04)$ \\
\hline $27-30$ & $1.17(0.71-1.92)$ & $0.44(0.26-0.72)^{*}$ \\
\hline $31-45$ & $0.92(0.53-1.59)$ & $0.28(0.16-0.49)^{*}$ \\
\hline \multicolumn{3}{|l|}{ Partner's Age (Ref 18-28) } \\
\hline $29-31$ & $0.74(0.48-1.13)$ & $0.88(0.58-1.35)$ \\
\hline $32-35$ & $0.73(0.44-1.20)$ & $1.09(0.66-1.80)$ \\
\hline $36-64$ & $0.56(0.29-1.09)$ & $1.58(0.81-3.07)$ \\
\hline \multicolumn{3}{|l|}{$\begin{array}{l}\text { Male Partner has other sexual } \\
\text { partners (Ref=No/Don't Know) }\end{array}$} \\
\hline Yes & $1.23(0.87-1.72)$ & - \\
\hline \multirow{2}{*}{\multicolumn{3}{|c|}{$\begin{array}{l}\text { Marital Status (Ref=Currently } \\
\text { Married) }\end{array}$}} \\
\hline & & \\
\hline Not Married & & $0.65(0.44-0.95)^{*}$ \\
\hline \multicolumn{3}{|l|}{ Partner Religion $($ Ref $=$ Catholic) } \\
\hline Protestant/Other Christian & $0.97(0.69-1.36)$ & $0.78(0.55-1.10)$ \\
\hline Other & $0.65(0.47-0.89)^{*}$ & $0.75(0.54-1.05)$ \\
\hline \multicolumn{3}{|l|}{$\begin{array}{l}\text { Woman's Education (Ref=Primary } \\
\text { or lower) }\end{array}$} \\
\hline Secondary or Higher & $0.75(0.56-0.996)^{*}$ & - \\
\hline \multicolumn{3}{|l|}{$\begin{array}{l}\text { Partner's Education (Ref=Primary } \\
\text { or lower) }\end{array}$} \\
\hline Secondary or Higher & $0.83(0.63-1.10)$ & $0.69(0.52-0.92)^{*}$ \\
\hline \multicolumn{3}{|l|}{$\begin{array}{l}\text { Food Insecurity ( } \operatorname{Ref}=\text { Always have } \\
\text { enough food and the kinds they } \\
\text { want) }\end{array}$} \\
\hline $\begin{array}{l}\text { Have enough food, but not alw } \\
\text { the kinds we want/Sometimes } \\
\text { don't have enough/Often don't } \\
\text { have enough }\end{array}$ & $0.82(0.62-1.08)$ & $0.76(0.57-1.005)$ \\
\hline \multicolumn{3}{|l|}{$\begin{array}{l}\text { Proportion of expenses met by } \\
\text { woman's earnings (Ref=None) }\end{array}$} \\
\hline Less than half & - & $1.39(0.51-3.79)$ \\
\hline Half & - & $1.43(0.55-3.71)$ \\
\hline More than half & - & $1.22(0.50-2.99)$ \\
\hline All & - & $2.31(0.94-5.65)$ \\
\hline \multicolumn{3}{|c|}{$\begin{array}{l}\text { Woman Baseline HIV Status } \\
\text { (Ref=Negative/Indeterminate/Don't } \\
\text { Know) }\end{array}$} \\
\hline Positive & $1.77(1.19-2.63)^{*}$ & - \\
\hline \multicolumn{3}{|l|}{ Facility (Ref=Nakaseke) } \\
\hline Entebbe & $0.75(0.53-1.06)$ & - \\
\hline $\begin{array}{l}\text { Mpigi } \\
* 95 \% \text { CI does not include } 1\end{array}$ & $1.11(0.76-1.64)$ & - \\
\hline
\end{tabular}


The significant sociodemographic variables for decision-making power included woman's age, marital status, and partner's education. Women who were $27-30$ or 31-45 were much less likely to have lower decision-making power compared to women who were 18-22 (OR $0.44,95 \%$ CI 0.26-0.72 and OR 0.28, 95\% CI 0.16-0.49, respectively). Those who were not married were less likely to have lower decision-making power compared to those who were currently married (OR 0.65, 95\% CI 0.44-0.95). Partners with a secondary or higher education were less likely to have lower decision-making power compared to partners with a primary or lower education (OR 0.69, 95\% CI 0.52-0.92).

\section{Discussion}

This analysis was conducted in order to determine the social and economic variables that were associated with gender inequality (as measured by attitudes towards intimate partner violence and decision-making power) among heterosexual couples expecting a child within the context of an HIV self-testing intervention trial in south-central Uganda. We found that Catholic religion, lower woman's education, and women with known HIV at baseline were associated with higher acceptance of intimate partner violence by the male partner. We also found that younger age of the woman, marital status of currently married, and lower partner's education was associated with lower decision-making power reported by the female partner.

Lower education was found to be associated with both higher acceptance of intimate partner violence and lower woman's decision-making power in our results. Similar results regarding education have been reported and shown not only for support for gender equality $8,9,23,24$, reproductive autonomy ${ }^{25}$, but also perpetration of intimate partner violence ${ }^{26-28}$, and we found similar results in our study in central Kenya among heterosexual couples expecting a child ${ }^{19}$.

In our analysis, we found that those reporting "Other/Not Christian" religion were less likely to have high acceptance of intimate partner violence compared to those who reported as being Catholic. These results are in the opposite direction from a study in Ghana that reported women who were Muslim and "Traditional" believers were more likely to approve domestic physical violence compared to women who were Christian ${ }^{11}$, and another study showing that compared to Catholic women, Muslims in Mali and Benin and followers of other religions in Zimbabwe were more likely to justify domestic abuse ${ }^{10}$. The male partner of women with a known history of HIV had significantly higher acceptance of intimate partner violence. This is in line from a study among married and cohabitating women in Zimbabwe that found that women had experienced any form of intimate partner violence were more likely to be HIV positive ${ }^{29}$, HIV positive women reported ${ }^{30}$, and in India, HIV positive women were three times more likely to experience sexual violence compared to HIV negative women ${ }^{31}$.

We found that currently married couples had lower decision-making power reported by the woman compared to couples who were cohabitating but unmarried. This is consistent with research in the Democratic Republic of the Congo, where they found that men who were unmarried or separated had higher support for gender equality than those who were married ${ }^{8}$. Younger women had lower decision-making power compared to older women in our analysis. This is in line with studies in Ghana, Ethiopia, and a literature review showing a negative association of women's age with both perpetration and justification of intimate partner violence (another measure of gender equality) ${ }^{11,26,32}$.

\section{Discussion}

This analysis was conducted in order to determine the social and economic variables that were associated with gender inequality (as measured by attitudes towards intimate partner violence and decision-making power) among heterosexual couples expecting a child within the context of an HIV self-testing intervention trial in south-central Uganda. We found that Catholic religion, lower woman's education, and women with known HIV at baseline were associated with higher acceptance of intimate partner violence by the male partner. We also found that younger age of the woman, marital status of currently married, and lower partner's education was associated with lower decision-making power reported by the female partner.

Lower education was found to be associated with both higher acceptance of intimate partner violence and lower woman's decision-making power in our results. Similar results regarding education have been reported and shown not only for support for gender equality ${ }^{8,9,23,24}$, reproductive autonomy ${ }^{25}$, but also perpetration of intimate partner violence ${ }^{26-28}$, and we found similar results in our study in central Kenya among heterosexual couples expecting a child ${ }^{19}$. 
In our analysis, we found that those reporting "Other/Not Christian" religion were less likely to have high acceptance of intimate partner violence compared to those who reported as being Catholic. These results are in the opposite direction from a study in Ghana that reported women who were Muslim and "Traditional" believers were more likely to approve domestic physical violence compared to women who were Christian ${ }^{11}$, and another study showing that compared to Catholic women, Muslims in Mali and Benin and followers of other religions in Zimbabwe were more likely to justify domestic abuse ${ }^{10}$. The male partner of women with a known history of HIV had significantly higher acceptance of intimate partner violence. This is in line from a study among married and cohabitating women in Zimbabwe that found that women had experienced any form of intimate partner violence were more likely to be HIV positive ${ }^{29}$, HIV positive women reported ${ }^{30}$, and in India, HIV positive women were three times more likely to experience sexual violence compared to HIV negative women ${ }^{31}$.

We found that currently married couples had lower decision-making power reported by the woman compared to couples who were cohabitating but unmarried. This is consistent with research in the Democratic Republic of the Congo, where they found that men who were unmarried or separated had higher support for gender equality than those who were married8. Younger women had lower decision-making power compared to older women in our analysis. This is in line with studies in Ghana, Ethiopia, and a literature review showing a negative association of women's age with both perpetration and justification of intimate partner violence (another measure of gender equality) $)^{11,26,32}$.

\section{Limitations}

There are a few notable limitations to this data. First, there were large proportions of missing data for the women's self-report of religion and employment status. This prevented us from using these variables in our multivariate analysis, and therefore we could have missed potential associations that we simply did not have the power to detect. Second, this data was analyzed in the context of an HIV self-testing intervention trial, so the population that was enrolled in this trial will not be generalizable to the general population of heterosexual couples expecting a child in this area due to the specific inclusion and exclusion criteria. Furthermore, gender inequality can only be interpreted in how it was measured (in this case, attitudes towards intimate partner violence and decision-making power), so we can only make interpretations within this context.

\section{Conclusion}

In summary, we found that lower women's age, lower man's and woman's education, being married, being Catholic, and the woman being HIV positive were associated with lower gender equality. This study helps to contribute to the body of literature regarding socio-demographic factors and gender inequality, especially in Uganda and in an HIV-related context in the setting of pregnancy. These results show some promising areas to target to potentially improve relationship gender equality (especially to increase education levels among both men and women, and attempt to reduce the HIV prevalence among women). These areas could also help create targeted interventions (specifically targeted in language and materials towards different religions or married couples) to improve gender equality in heterosexual couples expecting a child in Uganda.

\section{Acknowledgements}

The original data for this project was funded by the International Initiative for Impact Evaluation, grant agreement No. TW2.2.28. The project described was supported by the National Institutes of Health $\mathrm{Na}$ tional Center for Advancing Translational Sciences (NCATS) through Grant Numbers TL1 TR001451 \& UL1 TR001450, and by the Bill \& Melinda Gates Foundation [OPP1066935]. The original data for this project was funded by the International Initiative for Impact Evaluation, grant agreement No. TW2.2.28. The funding organizations had no role in the design of this study, current analysis, interpretation of data, or manuscript writing. The authors declare no conflicts of interest.

\section{References}

1. United Nations Women. Gender Mainstreaming Concepts and definitions [Internet]. [cited 2018 Dec 7]. Available from: http://www.un.org/womenwatch/osagi/conceptsandefinitions.htm

2. Organization for Social Science Research in Eastern and Southern Africa. Insights into Gender Equity, Equality, and Power Relations in Sub-Saharan Africa. Prah M, editor. Kampala: Fountain Publishers; 2013.

3. United National Development Programme. Human Development Report 2014. 2014.

4. Langen TT. Gender power imbalance on women's capacity to negotiate self-protection against HIV/ 
AIDS in Botswana and South Africa. Afr Health Sci. 2005;5(3):188-97.

5. Fladseth K, Gafos M, Newell ML, Mcgrath N. The Impact of Gender Norms on Condom Use among HIV-Positive Adults in KwaZulu-Natal, South Africa. PLoS One. 2015;10(4):122671.

6. Jewkes RK, Levin JB, Penn-Kekana LA. Gender inequalities, intimate partner violence and HIV preventive practices: findings of a South African cross-sectional study. Soc Sci Med. 2003;56:125-34 PubMed .

7. Miller CL, Bangsberg DR, Tuller DM, Senkungu J, Kawuma A, Frongillo EA, et al. Food Insecurity and Sexual Risk in an HIV Endemic Community in Uganda. AIDS Behav [Internet]. 2011 [cited 2018 Dec 7];15:1512-

9. Available from: https://www.ncbi.nlm.nih.gov/ pmc/articles/PMC3110536/pdf/10461_2010_Article_9693.pdf

8. Lusey H, San Sebastian M, Christianson M, Edin KE. Factors associated with gender equality among church-going young men in Kinshasa, Democratic Republic of Congo: a cross-sectional study. Int J Equity Health [Internet]. 2017 [cited 2018 Jul 25];16(1):213. Available from: http://www.ncbi.nlm.nih.gov/pubmed/29228996

9. Scott J, Hacker M, Averbach S, Modest AM, Cornish $\mathrm{S}$, Spencer D, et al. Influences of sex, age and education on attitudes towards gender inequitable norms and practices in South Sudan. Glob Public Health [Internet]. 2014 Aug 9 [cited 2018 Dec 7];9(7):773-86. Available from: http://www.ncbi.nlm.nih.gov/pubmed/25026024

10. Rani M, Bonu S, Diop-Sidibé N. An Empirical Investigation of Attitudes towards Wife-Beating among Men and Women in Seven Sub-Saharan African Countries. Afr J Reprod Health. 2004;8(3):116-36.

11. Doku DT, Asante KO. Women's approval of domestic physical violence against wives: analysis of the Ghana demographic and health survey. BMC Womens Health [Internet]. 2015 Dec 21 [cited 2018 Jul 25];15:120. Available from: http://www.ncbi.nlm.nih. gov/pubmed/26691763

12. Karamagi CA, Tumwine JK, Tylleskar T, Heggenhougen $\mathrm{K}$. Intimate partner violence against women in eastern Uganda: implications for HIV prevention. BMC Public Health [Internet]. 2006 Dec 20 [cited 2018 Dec 7];6(1):284. Available from: http://www.ncbi.nlm.nih. gov/pubmed/17116252

13. Amugsi DA, Lartey A, Kimani-Murage E, Mberu BU. Women's participation in household decision-making and higher dietary diversity: findings from nationally representative data from Ghana. J Heal Popul Nutr [Internet]. 2016 Dec 31 [cited 2019 Jan 30];35(1):16.
Available from: http://www.ncbi.nlm.nih.gov/pubmed/27245827

14. Adjiwanou V, Legrand T. Gender inequality and the use of maternal healthcare services in rural sub-Saharan Africa. Health Place [Internet]. 2014 [cited 2018 Nov 14];29:67-78. Available from: http://dx.doi. org/10.1016/j.healthplace.2014.06.001

15. Banda PC, Odimegwu CO, Ntoimo LFC, Muchiri E. Women at risk: Gender inequality and maternal health. Women Health [nternet]. 2017 [cited 2018 Nov 14];57(4):405-29. Available from: http://www.tandfonline.com/action/journalInformation?journalCode $=\mathrm{w}$ wah20

16. Singh K, Bloom S, Brodish P. Gender Equality as a Means to Improve Maternal and Child Health in Africa. Heal Care Women Int [Internet]. 2015 [cited 2018 Nov 14];36(1):57-69. Available from: https://www. ncbi.nlm.nih.gov/pmc/articles/PMC4333143/pdf/nihms657584.pdf

17. Corroon M, Speizer IS, Fotso J-C, Akiode A, Saad A, Calhoun L, et al. The role of gender empowerment on reproductive health outcomes in urban Nigeria. $\mathrm{Ma}$ tern Child Health J [Internet]. 2014 Jan [cited 2018 Dec 7];18(1):307-15. Available from: http://www.ncbi.nlm. nih.gov/pubmed/23576403

18. Mohammed BH, Johnston JM, Harwell JI, Yi H, Tsang KW, Haidar JA. Intimate partner violence and utilization of maternal health care services in Addis Ababa, Ethiopia. BMC Health Serv Res [Internet]. 2017 Dec 7 [cited 2018 Dec 7];17(1):178. Available from: http://www.ncbi.nlm.nih.gov/pubmed/28270137

19. Vrana-Diaz CJ, Korte JE, Gebregziabher M, Richey L, Selassie A, Sweat M, et al. Social and Demographic Predictors of Gender Inequality Among Heterosexual Couples Expecting a Child in Central Kenya. Glob Soc Welf. 2019;

20. Compendium of Gender Scales. Gender Equitable Men (GEM) Scale [Internet]. [cited 2017 Apr 17]. Available from: https://www.c-changeprogram.org/ content/gender-scales-compendium/gem.html

21. Agresti A. Categorical data analysis [Internet]. 3rd ed. Hobboken, NJ: John Wiley \& Sons; 2013 [cited 2018 Aug 20]. 714 p. Available from: https://www.wiley.com/ en-us/Categorical+Data + Analysis $\% 2 C+3 r d+$ Edition-p-9780470463635

22. Breslow NE, Clayton DG. Approximate Inference in Generalized Linear Mixed Models. J Am Stat Assoc [Internet]. 1993 Mar [cited 2018 Aug 20];88(421):9. Available from: https://www.jstor.org/stable/2290687?origin $=$ crossref

23. Levtov RG, Barker G, Contreras-Urbina M, 
Heilman B, Verma R. Pathways to Gender-equitable Men: Findings from the International Men and Gender Equality Survey in Eight Countries. 2014 [cited 2018 Jul 25];17(5):467-501. Available from: http://journals. sagepub.com/doi/pdf/10.1177/1097184X14558234

24. Slegh H, Barker G, Levtov R. Gender Relations, Sexual and Gender-Based Violence and the Effects of Conflict on Women and Men in North Kivu, Eastern Democratic Republic of the Congo: Results from the International Men and Gender Equality Survey (IMAGES) [Internet]. Washington, DC; Capetown, South Africa; 2014 [cited 2018 Jul 25]. Available from: http:/ / www.partners4prevention.org/

25. Princewill CW, De Clercq E, Riecher-Rössler A, Jegede AS, Wangmo T, Elger BS. Education and Reproductive Autonomy: The Case of Married Nigerian Women. Narrat Inq Bioeth. 2017;7(3):231-44.

26. Capaldi DM, Knoble NB, Shortt JW, Kim HK. A Systematic Review of Risk Factors for Intimate Partner Violence. Partner Abuse [Internet]. 2012 Apr [cited 2018 Jul 25];3(2):231-80. Available from: http://www.ncbi. nlm.nih.gov/pubmed/22754606

27. Osinde MO, Kaye DK, Kakaire O. Intimate partner violence among women with HIV infection in rural Uganda: critical implications for policy and practice. BMC Womens Health [Internet]. 2011 Nov 17 [cited 2018 Jul 25];11:50. Available from: http://www.ncbi.nlm.nih. gov/pubmed/22093904
28. Vakili M, Nadrian H, Fathipoor M, Boniadi F, Mohammad BS, Morowatisharifabad A. Prevalence and Determinants of Intimate Partner Violence Against Women in Kazeroon, Islamic Republic of Iran. Violence Vict [Internet]. 2010 [cited 2018 Jul 26];25(1):116. Available from: https://musc-illiad-oclc-org.ezproxy-v. musc.edu/illiad/illiad.dll?Action $=10 \&$ Form $=75 \&$ Value $=46403$

29. Henderson L, Zerai A, L. Morrow R. Intimate Partner Violence and HIV Status among Ever-Married and Cohabiting Zimbabwean Women: An Examination of Partners' Traits. Afr J Reprod Health [Internet]. 2017 Dec 30 [cited 2018 Dec 7];21(4):45-54. Available from: http://www.ncbi.nlm.nih.gov/pubmed/29624950

30. Rwafa T, Shamu S, Christofides N. Relationship power and HIV sero-status: An analysis of their relationship among low-income urban Zimbabwean postpartum women. BMC Public Health. 2019 Jun 21;19(1).

31. Chakraborty H, Patted S, Gan A, Islam F, Revankar A. Determinants of Intimate Partner Violence Among HIV-Positive and HIV-Negative Women in India. J Interpers Violence [Internet]. 2016 Feb 6 [cited 2018 Jul 25];31(3):515-30. Available from: http://www.ncbi. nlm.nih.gov/pubmed/25381267

32. Gurmu E, Endale S. Wife beating refusal among women of reproductive age in urban and rural Ethiopia. BMC Int Health Hum Rights [Internet]. 2017 [cited 2018 Dec 7];17(1):6. Available from: http://www.ncbi. nlm.nih.gov/pubmed/28302105 